\title{
Intrauterine exposure to maternal stress alters $B d n f$ DNA methylation and telomere length in the brain of adult rat offspring
}

\author{
Jennifer Blaze ${ }^{1}$, Arun Asok ${ }^{1}$, Kristyn Borrelli ${ }^{1}$, Christine Tulbert $^{2}$, Justin Bollinger ${ }^{2}$, \\ April E. Ronca ${ }^{2-4}$, \& Tania L. Roth*1 \\ ${ }^{1}$ Department of Psychological and Brain Sciences, University of Delaware, Newark, DE \\ ${ }^{2}$ Obstetrics and Gynecology, ${ }^{3}$ Program in Neuroscience, ${ }^{4}$ Molecular Medicine \& Translational \\ Science, Wake Forest School of Medicine, Winston-Salem, NC. \\ *Denotes equal contributions
}

Address correspondence to: Tania L. Roth, Ph.D., Department of Psychological and Brain Sciences, University of Delaware, 108 Wolf Hall, Newark, DE 19716. phone - (302) 831-2787, email - troth@psych.udel.edu

Jennifer Blaze current address:

Department of Neuroscience, Icahn School of Medicine at Mt. Sinai, New York, NY

Arun Asok current address:

Department of Neuroscience, Columbia University, New York, NY

Howard Hughes Medical Institute, New York, NY

Justin Bollinger current address:

Department of Psychological and Brain Sciences, Indiana University, Bloomington, IN 


\begin{abstract}
DNA methylation (addition of methyl groups to cytosines which normally represses gene transcription) and changes in telomere length (TTAGGG repeats on the ends of chromosomes) are two molecular modifications that result from stress and could contribute to the long-term effects of intrauterine exposure to maternal stress on offspring behavioral outcomes. Here, we measured methylation of Brain-derived neurotrophic factor (Bdnf), a gene important in development and plasticity, and telomere length in the brains of adult rat male and female offspring whose mothers were exposed to unpredictable and variable stressors throughout gestation. Males exposed to prenatal stress had greater methylation (Bdnf $I V)$ in the medial prefrontal cortex (mPFC) compared to non-stressed controls. Further, prenatally-stressed males had shorter telomeres than controls in the mPFC. This study provides the first evidence in a rodent model of an association between prenatal stress exposure and subsequent shorter brain telomere length. Together findings indicate a long-term impact of prenatal stress on DNA methylation and telomere biology with relevance for behavioral and health outcomes, and contribute to a growing literature linking stress to intergenerational epigenetic alterations and changes in telomere length.
\end{abstract}

Keywords: prenatal stress, maternal stress, DNA methylation, telomere length 


\section{Introduction}

Perturbations during gestation in the form of malnutrition, infection, or psychosocial stress have devastating consequences on the physical and mental health well-being of offspring. For example, people exposed to famine during gestation (such as that of the Dutch Famine of 1944) have significant physical health problems including cardiovascular disease and high blood pressure (Painter et al., 2008; Roseboom et al., 2011). Gestational famine exposure also produces long-lasting neurobiological and behavioral consequences, including an increased incidence of schizophrenia (Hoek et al., 1996; Roseboom et al., 2011). Psychosocial stress experienced by the mother during gestation likewise produces vulnerability to psychopathology in offspring, including increased risk for schizophrenia and depression (Betts et al., 2015; Malaspina et al., 2008). Further, work from various animal models provides additional evidence that a suboptimal intrauterine environment has long-term physical and behavioral consequences for offspring (for reviews see (Bock et al., 2015; Driscoll and Barr, 2016; Lupien et al., 2009)).

One way that prenatal stress could affect brain function and behavior is through epigenetic modifications, including DNA methylation. DNA methylation is characterized by the addition of methyl groups to cytosines, typically of cytosine-guanine dinucleotides (CG sites), which can dampen transcriptional activity through the blockade of transcription factors or the recruitment of transcriptional silencing machinery. Recent studies have also highlighted the role of active DNA demethylation in regulating transcription within the CNS, and candidates thought to reverse DNA methylation include ten-eleven-translocation (TET) proteins (Kohli and Zhang, 2013), GADD45b (Ma et al., 2009), and MBD2 (Detich et al., 2002). Though traditionally attributed to early cell development and differentiation, there is now a vast literature showing that DNA methylation/demethylation remains an active process even occurring in mature neurons. Further, studies in humans (Cao-Lei et al., 2014; Kundakovic and Champagne, 2011; 
Nye et al., 2014) and animals (Boersma et al., 2014; Dong et al., 2015; Jensen Peña et al., 2012; Matrisciano et al., 2013; Morgan and Bale, 2011; Mueller and Bale, 2007, 2008) have highlighted the ability of prenatal stress to alter DNA methylation at various gene loci, including the Brain-derived neurotrophic factor (Bdnf) gene. Bdnf is crucial for development and plasticity in the developing and adult brain and is implicated in various psychiatric disorders, including depression, schizophrenia, and PTSD (Martinowich et al., 2007). Given the widespread functioning of $B d n f$ across brain regions throughout early development, this locus may play a role in translating prenatal stress to adult phenotypic outcomes including psychopathology.

Recent work has also sought to characterize the detrimental impact of early-life stress by examining changes in telomere length -stretches of TTAGGG repeats located on the ends of chromosomes (for a comprehensive review, see (Blaze et al., 2015)). Studies continue to show that various forms of early-life stress (e.g., perinatal complications, psychological stress, physical abuse, etc.) during the prenatal (Entringer et al., 2011; Entringer et al., 2013; Shalev et al., 2014a) or postnatal (Asok et al., 2013; Kananen et al., 2010; Kiecolt-Glaser et al., 2011; O'Donovan et al., 2011; Tyrka et al., 2010) period shorten telomere length in the periphery. Given that telomeres are dynamically regulated during the first years of life (Slagboom et al., 1994; Zeichner et al., 1999), examining neural changes in telomere length following prenatal stress is another important avenue for understanding how stress in early-life may relate to future mental well-being.

In daily life, humans tend to be exposed to unpredictable challenges that vary in magnitude and duration. Studies of prenatal stress in rodents often employ repeated presentations of a single, non-varying stressor, most frequently, immobilization stress (de Souza et al., 2013; Jensen Peña et al., 2012; Matrisciano et al., 2013). However rodent studies provide evidence for 
habituation to predictable, non-varying stressors (Armario, 2006; Daviu et al., 2014), and studies employing variable prenatal stress paradigms have reported etiological changes that may be more closely linked to psychiatric disorders (Bock et al., 2015; Mueller and Bale, 2008; Sickmann et al., 2015; Wilson et al., 2012). Collectively, these findings suggest that variable, unpredictable stress may model the human condition with greater fidelity than invariant stress paradigms.

The current study was designed to characterize changes in Bdnf DNA methylation and telomere length across multiple discrete brain regions of the adult rat following unpredictable variable prenatal stress (UVPS). We chose to focus on the medial prefrontal cortex, ventral hippocampus, dorsal hippocampus, and central/basolateral amygdala, as our prior work showed changes in Bdnf exon IV DNA methylation and telomere length in these regions in adult animals subjected to maternal maltreatment (Asok et al., 2014; Blaze et al., 2013; Roth et al., 2009; Roth et al., 2014). Changes in Bdnf IV DNA methylation have likewise been found in these same brain regions in other models of prenatal stress (Boersma et al., 2014; Dong et al., 2015).

\section{Materials and Methods}

\subsection{Subjects}

Female Sprague-Dawley (SD) rats and their offspring were used ( $\mathrm{n}=6$ control animals per sex, 7 UVPS animals per sex derived from 13 dams, with only one male and one female offspring from each control and UVPS litter). To avoid stress associated with shipping during pregnancy, dams were time-mated in our breeding colony (Taconic Farms, Germantown, NY). Pregnancy was determined by daily vaginal lavage with the presence of spermatozoa indicating Gestational day zero (G0). Throughout pregnancy, dams were housed in groups of three in 
standard maternity cages $(47 \mathrm{~cm} \times 26 \mathrm{~cm} \times 21 \mathrm{~cm})$ lined with corncob bedding and maintained under standard colony conditions (12:12 light/dark cycle [0600:1800]; $21+/-1^{\circ} \mathrm{C}$ at $30-50 \%$ humidity). Rat chow (Purina \#5102) and water were available ad libitum. On gestational day (G) 21, dams were singly housed. Animal experimentation was conducted in full accordance with the NRC Guide for the Care and Use of Laboratory Animals (National Academy of Sciences, copyright 2011). All procedures were approved by the Wake Forest School of Medicine Animal Care and Use Committee (ACUC).

\subsection{Unpredictable, Variable Prenatal Stress (UVPS) Paradigm}

On G0, pregnant dams were randomly assigned to either control or UVPS treatment conditions (Body weight: CNTL, $288 \pm 26 \mathrm{~g}$; UVPS, $292 \pm 23 \mathrm{~g}$ ). On G1, UVPS dams were exposed daily to three stressors, one during each of three discrete time periods varying in duration (Time periods, Early: 0600-1200, Mid: 1200-1800, Late: 1800-600; Duration, 15, 30 or 60 minutes). Stressors included tube restraint (PVC tube, length $\mathrm{x}$ diameter: $21.6 \times 6.4 \mathrm{~cm}$ ), high-frequency strobe light $(60 \mathrm{~Hz}, 3.5 \mathrm{~W}, 85 \mathrm{~mA})$, and white-noise induced stress $(80 \mathrm{db})$. Stimulus presentation was block randomized across pregnancy and spanned G1 - G21. Control dams were handled briefly each day to match UVPS handling. To eliminate potential confounds of altered postnatal maternal care by UVPS dams contributions on experimental endpoints, offspring in both conditions were fostered at birth to non-manipulated, newly parturient dams. 


\subsection{Tissue Collection and Processing}

On postnatal day (PN) 90, rats were euthanized and flash frozen. Brain were extracted and later sectioned into $300 \mu \mathrm{m}$ slices on a cryostat. DNA and RNA were simultaneously extracted from homogenized tissue punches of medial prefrontal cortex (mPFC; consisting of infralimbic and prelimbic cortices), hippocampus (dorsal vs. ventral) and central/basolateral amygdala (Qiagen Inc., Valencia, CA). Quantity and quality of nucleic acids were determined using spectrophotometry (Nanodrop 2000) and then frozen at $-80^{\circ} \mathrm{C}$ for later processing.

\subsection{Bdnf DNA Methylation and Gene Expression Assays}

For methylation assays, DNA was bisulfite-converted (Epitect Bisulfite Kit, Qiagen, Inc., Valencia, CA) and direct bisulfite-sequencing PCR (BSP) was used to measure methylation at 11 CG sites in Bdnf exon IV as previously reported (Roth et al., 2009; Roth et al., 2014). PCR products were purified (Diffininty Genomics, RapidTip) and sequenced using reverse primers at the Delaware Biotechnology Institute. The electropherogram was read on Chromas software, where the percent methylation of each CG site was determined by the ratio between peak values of $\mathrm{G}$ and $\mathrm{A}(\mathrm{G} /[\mathrm{G}+\mathrm{A}])$. We confirmed the accuracy of this technique by assessing methylated standards (Rat Pre-mixed Calibration Standards, Epigendx) ranging from 0-100\% methylation and performing a linear regression on methylation values $\left(\mathrm{F}(1,40)=228.9, \mathrm{p}<0.001, \mathrm{r}^{2}=0.8512\right)$.

For gene expression assays, RNA was reverse transcribed using a cDNA synthesis kit (Qiagen, Inc., Valencia, CA) and amplified via real-time PCR using Taqman probes (ThermoFisher Scientific, Grand Island, NY) to target Bdnf exon IV-containing transcripts. Tubulin was used as a reference gene and all reactions were run in triplicate. The comparative $\mathrm{Ct}$ 
method was used to obtain the relative $B d n f I V$ mRNA fold change in experimental (UVPS) relative to control animals (Livak and Schmittgen, 2001).

\subsection{Telomere Length and Telomerase assays}

DNA was assessed for purity, and diluted to 10ng/uL via Nanodrop spectrophotometry following procedures used previously(Asok et al., 2014). Quantitative real-time PCR was conducted with the following primers targeting telomeres (T; Forward ${ }_{\mathrm{TEL}}: 5^{\prime}$ CGGTTTGTTTGGGTTTGGGTTTGGGTTTGGGTTTGGGTT-3’' and Reverse ${ }_{\mathrm{TEL}}$ :5'GGCTTGCCTTACCCTTACCCTTACCCTTACCCTTACCCT-3') and a single copy control

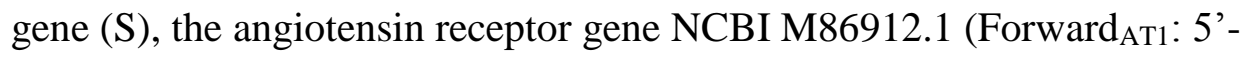
ACGTGTCTCAGCATCGACCGCTACC -3' and Reverse ${ }_{\mathrm{AT} 1}$ : 5'-

AGAATGATAAGGAAAGGGAACAAGAAGCCC -3') (O'Callaghan and Fenech, 2011). Each PCR well contained a final concentration of 1x Power Sybr Green Master Mix (ThermoFisher Scientific, Grand Island, NY), $100 \mathrm{nM}$ forward primer, $100 \mathrm{nM}$ reverse primer, and 20ng of sample DNA. The experimenter was blind to a sample's group identification when loading the PCR plate. For each sample, the telomere and AT1 qPCR assays were carried out in triplicate in the same well position on different 96-well plates. All male and female samples for a particular brain region were run on the same 96-well PCR plate. Given that plates did not contain a standard, we did not statistically contrast brain regions across PCR plates. All samples were assayed in triplicate and any replicate that deviated \pm 1 cycle threshold $(\mathrm{Ct})$ beyond the triplicate average ( $\sim 4.3 \%$ of all replicates) was excluded from the final $\mathrm{Ct}$ average. Eight samples failed to amplify in the amygdala ( 2 females and 2 males in the control condition and 1 female and 3 
males in the UVPS condition) and were thus excluded from analyses. Relative telomere length was calculated as a ratio of telomeres $(\mathrm{T})$ to single copy gene $(\mathrm{S})$ by the formula $\mathrm{T} / \mathrm{S}=\left(2^{\Delta \mathrm{Ct}}\right.$ $\left.{ }^{\text {tel }}\right) /\left(2^{\Delta \mathrm{CtAT} 1}\right)$. The $\mathrm{T} / \mathrm{S}$ ratio was then transformed into a proportionate score against the unstressed control condition for each brain region.

Similar to $B d n f I V$ gene expression assays, cDNA was amplified via real-time PCR using Taqman probes (ThermoFisher Scientific, Grand Island, NY) that targeted telomerase exons 7-9containing transcripts. Tubulin was also used as a reference gene and all reactions were run in duplicate. The comparative $\mathrm{Ct}$ method was used to obtain the relative mRNA fold change in experimental (UVPS) relative to control animals (Livak and Schmittgen, 2001).

\subsection{Statistical Analyses}

For each brain region, BSP data were analyzed with two-way ANOVAs comparing average methylation (across all 11 sites) between treatment group and sex ( 2 x 2), as well as twoway ANOVAs on each of the $11 \mathrm{CG}$ sites [treatment group (2) x sex (2)]. Telomere length between conditions and sexes was likewise analyzed with two-way ANOVAs for each brain region. Bonferroni multiple comparison tests were used when appropriate for post hoc analyses. Differences in Bdnf or telomerase mRNA levels were analyzed by one-sample t-tests (for comparison of UVPS subjects to controls). Differences were considered to be statistically significant for $\mathrm{p} \leq 0.05$, and non-significant trends at $\mathrm{p} \leq 0.1$ are also reported. 


\section{Results}

\subsection{Bdnf IV DNA methylation}

To assess the effects of UVPS on adult DNA methylation patterns, we measured methylation of Bdnf IV in adult (PN90) male and female rats. The primer set we used targeted DNA that encompasses a transcription factor binding and transcription start site. We targeted this region as there are ample data indicating that this region of the $B d n f$ gene seems especially sensitive to a range of environmental (physical or psychosocial) factors (Kundakovic et al., 2013; Lubin et al., 2008; Roth et al., 2009; Roth et al., 2014; Roth et al., 2011).

Medial prefrontal cortex ( $m P F C$ ). UVPS males had higher levels of methylation in the mPFC (Figure 1A). A two-way ANOVA comparing average methylation values for both treatment (UVPS vs. control) and sex (males vs. females) revealed a significant interaction $(\mathrm{F}(1,19)=18.19, \mathrm{p}=0.0004)$. UVPS males had higher overall methylation than control males $(\mathrm{t}=3.512, \mathrm{p}=0.0140)$ and UVPS females $(\mathrm{t}=3.374, \mathrm{p}=0.0191)$. UVPS males also had higher methylation at several individual CG sites. A two-way ANOVA comparing methylation values at CG site 1 for both treatment (UVPS vs. control) and sex (males vs. females) revealed a significant interaction $(\mathrm{F}(1,19)=16.66, \mathrm{p}=0.0006)$. UVPS males had higher methylation than control males ( $\mathrm{t}=3.165, \mathrm{p}=0.0306$ ) and UVPS females $(\mathrm{t}=3.552, \mathrm{p}=0.0128)$. A two-way ANOVA comparing methylation values at CG site 2 for both treatment and sex revealed a significant interaction $(\mathrm{F}(1,19)=12.31, \mathrm{p}=0.0023)$. Control females had marginally higher methylation than UVPS females ( $\mathrm{t}=2.854, \mathrm{p}=0.0609)$ and control males $(\mathrm{t}=2.794, \mathrm{p}=0.0694)$. A two-way ANOVA comparing methylation values at CG site 3 for both treatment and sex revealed a significant interaction $(\mathrm{F}(1,19)=16.75, \mathrm{p}=0.0006)$. UVPS males had higher methylation than control males 
$(\mathrm{t}=2.921, \mathrm{p}=0.0525)$ and UVPS females $(\mathrm{t}=3.366, \mathrm{p}=0.0195)$. A two-way ANOVA comparing methylation values at CG site 4 for both treatment and sex revealed a significant main effect of treatment $(\mathrm{F}(1,17)=13.75 \mathrm{p}=0.0017)$, sex $(\mathrm{F}(1,17)=6.453, \mathrm{p}=0.0211)$, and an interaction $(\mathrm{F}(1,17)=25.35, \mathrm{p}=0.0001)$. UVPS males had higher methylation than control males $(\mathrm{t}=6.052$, $\mathrm{p}<0.0001)$, control females $(\mathrm{t}=4.325, \mathrm{p}=0.0028)$, and UVPS females $(\mathrm{t}=5.477, \mathrm{p}=0.0002)$. Finally, a two-way ANOVA comparing methylation values at CG site 7 for both treatment and sex revealed a significant interaction $(\mathrm{F}(1,19)=14.38, \mathrm{p}=0.0012)$. UVPS males had higher methylation than control males $(\mathrm{t}=3.452, \mathrm{p}=0.0160)$ and UVPS females $(\mathrm{t}=3.145, \mathrm{p}=0.0320)$.

Ventral hippocampus. As depicted in Figure 1B UVPS females had somewhat higher levels of methylation for several CG sites, though most statistical comparisons failed to reach significance. A two-way ANOVA comparing average methylation values for both treatment (UVPS vs. control) and sex (males vs. females) revealed a marginally significant interaction with females showing higher overall methylation $(\mathrm{F}(1,22)=3.917, \mathrm{p}=0.0605)$. A two-way ANOVA comparing methylation values at individual sites for both treatment (UVPS vs. control) and sex (males vs. females) revealed a significant interaction at $\mathrm{CG}$ site $1(\mathrm{~F}(1,22)=5.672, \mathrm{p}=0.0263)$ and CG site $2(\mathrm{~F}(1,21)=4.935, \mathrm{p}=0.0374)$, though all post hoc analyses failed to reach statistical significance (for site 1 however the comparison between control and UVPS females reached marginal significance $(\mathrm{t}=2.607, \mathrm{p}=0.0966))$.

Dorsal hippocampus and amygdala. There were no main effects of treatment or sex, nor any interaction effects for average methylation levels or methylation at individual CG sites within the dorsal hippocampus or central/basolateral amygdala. 


\subsection{Bdnf IV gene expression}

As one way to envision the functional relevance of our changes in DNA methylation induced by UVPS within the mPFC, we measured steady-state gene expression of Bdnf exon IVcontaining transcripts. No significant differences in Bdnf IV mRNA levels were detected between UVPS males and controls $(\mathrm{t}(6)=1.900, \mathrm{p}=0.1061)$ nor UVPS females and controls

$(\mathrm{t}(6)=1.693, \mathrm{p}=0.1415)$. These data however do not necessarily mean our observed methylation changes are not functionally relevant in terms of Bdnf gene expression. DNA methylation changes do not always result in steady-state changes in gene expression, but can instead prime transcriptional responses to subsequent stimuli and neural activation (Baker-Andresen et al., 2013).

\subsection{Telomere length}

Medial prefrontal cortex ( $M P F C$ ) telomere length. UVPS had a significant effect on telomere length in the mPFC (Figure 2). A two-way ANOVA revealed a main effect of treatment (UVPS vs. control) on telomere length in the $\mathrm{mPFC}(\mathrm{F}(1,22)=7.847, \mathrm{p}=0.0104)$. UVPS males exhibited shorter telomeres than control males $(\mathrm{t}(11)=2.230, \mathrm{p}=0.048)$. In females, UVPS females exhibited marginally shorter telomeres relative to controls $(t(11)=1.902, p=0.084)$. Ventral hippocampus, dorsal hippocampus, and amygdala telomere length. In the hippocampus and central/basolateral amygdala there were no main effects of treatment or sex, nor any interaction effects on telomere length. 


\subsection{Medial prefrontal cortex ( $m P F C$ ) telomerase $m R N A$}

Given the differences observed in telomere length within the mPFC, we also wanted to selectively examine telomerase in this brain region. No significant differences in telomerase were detected between UVPS males vs. controls $(\mathrm{t}(6)=1.807, \mathrm{p}=0.120)$, but there was a marginal decrease in telomerase mRNA for UVPS females compared to control females $(\mathrm{t}(6)=2.265$, $\mathrm{p}=0.064)$.

\section{Discussion}

Here we used a rodent model of unpredictable, variable prenatal stress to test the hypothesis that a stressful environment in utero can render epigenetic and telomeric changes in the brain of offspring. We observed that $B d n f I V$ DNA methylation patterns in offspring at age PN90 varied depending on prenatal condition, sex, and brain region. Specifically, males from prenatally stressed mothers had significantly higher levels of methylation in the mPFC compared to controls. This effect was not present in females from stressed mothers, who instead had lower levels of methylation than prenatally stressed males within the mPFC and marginally higher levels of methylation than controls within the ventral hippocampus. No differences in $B d n f$ IV DNA methylation were found in the amygdala or dorsal hippocampus in the stressed group compared to controls for either sex. Similarly brain-region specific, stress-induced effects on telomere length were detected - male, and to a lesser extent female, UVPS offspring exhibited shorter telomeres in the mPFC with no differences in the amygdala or dorsal hippocampus.

While decades of research have shown the detrimental effects of prenatal stress on brain function and behavior, there remains much to elucidate regarding the molecular mechanisms underlying these effects. A large drawback of this field as a whole is the variance in prenatal 
stress paradigms used, which makes direct comparisons between studies difficult (Bock et al., 2015). The timing of gestational stress, predictability of stressor, and the stressors used vary greatly between studies, but are extremely important factors when considering the neurobiological changes induced by prenatal stress.

Studying PN21 and PN80 male offspring of pregnant rats subjected to a different variable stress paradigm (beginning on G14, dams were subjected to a mixture of restraint stress, forced swim, cold exposure, social stress, and light exposure overnight) than the one we employed here, another lab found that prenatally stressed males at both ages had higher Bdnf IV DNA methylation in the amygdala and hippocampus (only a site-specific change at PN80) (Boersma et al., 2014). One CG site showed higher methylation within the PFC of prenatally stressed males at PN21, however no methylation differences were detected in the PFC of PN80 offspring (Boersma et al., 2014). Studying PN75 male offspring of pregnant mice subjected to restraint stress under bright light conditions (45 minutes three times per day from G7 through delivery), another lab observed significantly higher levels of methylation (as well as hydroxymethylation) for DNA associated with Bdnf $I V$ in the frontal cortex (Dong et al., 2015). Finally, studying PN90 male and female offspring of pregnant mice exposed to predator stress (from G11-18 dams were daily exposed to three different predator urines), another lab found lower methylation of $B d n f I V$ DNA (at only one CG site) in the hippocampus of female offspring (St-Cyr and McGowan, 2015).

Differences between these studies and ours may be attributable to differences in the type and timing of prenatal stress. Our study is unique in that we employed a block randomized program of three stressors (light, sound and restraint) presented early- and mid-day during the lights on phase of the cycle and once during the dark phase for varying durations ranging from 
15 to 60 minutes for the entire span of gestation. In addition, examining methylation within the entire prefrontal cortex, amygdala, and hippocampus instead of the specific divisions within could produce different results. Nonetheless, data altogether are consistent with the notion that stress experienced by the dam is able to produce long-term epigenetic changes in offspring at a gene well-linked to neuroplasticity and cognition (e.g.(Bekinschtein et al., 2008; Dincheva et al., 2016; Martinowich et al., 2007; McEwen et al., 2015)) and mental health (e.g.(Fuchikami et al., 2011; Keller et al., 2010; Perroud et al., 2013; Smith et al., 2011)). Our study and that from the McGowan lab shed light on the sex-specific epigenetic changes inducible by intrauterine exposure to maternal stress.

Here we also found that prenatally stressed males exhibited shorter telomeres in the medial prefrontal cortex compared to controls. A number of studies have demonstrated a strong relationship between early-life stress, reduced peripheral telomere length, and future mentalhealth (Entringer et al., 2011; Marchetto et al., 2016; Shalev et al., 2014b). The effects of stress on telomere length in the brain is less clear (Thomas et al., 2008). Telomeres shorten with age in the cortex of rodents, but this shortening is thought to reflect changes occurring within glial cells given their increased mitotic potential relative to neurons (Flanary and Streit, 2003; Linkus et al., 2016; Szebeni et al., 2014). We did not distinguish between neurons and glia in measuring telomere length or methylation in the present study, although recent studies from our lab suggest that developmental (postnatal) stress induces changes in mPFC Bdnf IV DNA methylation primarily in neurons (Blaze and Roth, 2017).

Rats selectively bred for enhanced depressive-like symptoms (i.e., the Flinders-sensitive line) show reduced telomere length, telomerase, and $B d n f$ expression in the hippocampus (Wei et al., 2015) - supporting a role for telomere shortening in the brain. In contrast, other studies have 
found that postnatal stress (e.g., maternal separation or caregiver maltreatment) is associated with longer telomeres in the ventral hippocampus and medial prefrontal cortex (Asok et al., 2014; Botha et al., 2012). The findings here complement our previous work with regard to stress effects on a common brain region (i.e., the medial prefrontal cortex), but differ in the direction of telomere change. Similar to methylation, it is likely that the type (maltreatment vs. maternal separation vs. variable stress) and timing (pre- vs. postnatal) of stress can differentially affect brain telomere length, and future studies are certainly warranted to better understand the parameters affecting brain telomere length.

In summary our findings highlight the capacity of in utero stress to leave its epigenetic and telomeric mark within specific regions of the brain, providing important new insights into how gestational stress may have far reaching behavioral consequences for progeny. Indeed, behavioral analysis of the prenatally stressed and non-stressed subjects used in this report revealed robust sex-specific (male-biased) effects on anxiety responses (Ronca, unpublished). These findings are consistent with reports that prenatal stress leads to heightened stress sensitivity, especially among males (Bronson and Bale, 2014), including studies employing variable prenatal stress paradigms (Koenig et al., 2005; Wilson et al., 2013). In accord with previous research linking prenatal stress with changes in body weight regulation and metabolic disorders (Boersma et al., 2014; Wilson et al., 2013), adult body weights of prenatally stressed male (but not female) offspring of the present study were significantly elevated relative to nonstressed offspring (Ronca, unpublished). Our findings indicate that, in addition to brain epigenetic and telomeric changes, unpredictable, variable gestational stress exerts clear sexspecific programming effects on the physical and behavioral health of the offspring. 
The study reported herein provides the first evidence in a rodent model for an enduring influence of prenatal stress on both DNA methylation and telomere length in adulthood. Going forward, the challenge will be to identify the specific role(s) of these molecular brain changes in relation to the diverse effects of early life stressors. Recent progress characterizing physiological and structural changes in the brain associated with prenatal stress, and their sex-specificity, may provide important new insights. Neuronal changes are highly region-specific with the most dramatic changes occurring in limbic and prefrontal cortical areas, those involved in cognition and emotional behavior. Altered neurogenesis, neuronal arborization, neuronal density, dendritic architecture, synaptic connectivity, and myelination have all been reported in animal models of prenatal stress (Bock et al., 2015; Schuurmans and Kurrasch, 2013; Soztutar et al., 2016). Telomere length may be related to changes in a variety of cells throughout the brain, including glia (Dabouras et al., 2004; Leventopoulos et al., 2007). Relating these cellular alterations to epigenetic and telomeric changes in common brain regions will likely lead to deeper understanding of the underlying mechanisms.

While extensive progress has been made in the understanding of the role of early life adversity in the development of psychiatric and metabolic disorders, more detailed research on sex-specific epigenetic brain structural and behavioral changes is warranted. In particular, consistency across gestational stress paradigms, including the predictability and timing of stressor, the use of common behavioral and endpoint measures, and comparisons between male and females are vital for uncovering consistent and meaningful patterns of neurobiological changes induced by prenatal stress. Regardless, our present findings have strong relevance for behavioral and health outcomes, and contribute to a growing literature linking stress to early developmental programming, intergenerational epigenetic transmission of environmental 
exposures, and telomere biology. Illuminating molecular mechanisms underlying transgenerational programming of stress responses and mental and physical health outcomes are important first steps toward establishing therapeutic interventions to halt the generational persistence of pathological outcomes associated with early life adversity. 
Acknowledgments: This research was supported by NIGMS (1P20GM103653) and private donation funds to TLR and NICHD (1R01HD50201) to AER. We thank the Delaware Biotechnology Institute for the use of their core facilities (supported by the Delaware INBRE program, with a grant from NIGMS (P20 GM103446) and the state of Delaware). 


\section{References}

Armario, A., 2006. The hypothalamic-pituitary-adrenal axis: what can it tell us about stressors? CNS \& Neurological Disorders Drug Targets 5, 485-501.

Asok, A., Bernard, K., Rosen, J.B., Dozier, M., Roth, T.L., 2014. Infant-caregiver experiences alter telomere length in the brain. PLoS One 9, e101437.

Asok, A., Bernard, K., Roth, T.L., Rosen, J.B., Dozier, M., 2013. Parental responsiveness moderates the association between early-life stress and reduced telomere length. Development and Psychopathology 25, 577-585.

Baker-Andresen, D., Ratnu, V.S., Bredy, T.W., 2013. Dynamic DNA methylation: a prime candidate for genomic metaplasticity and behavioral adaptation. Trends in Neurosciences 36, 313.

Bekinschtein, P., Cammarota, M., Izquierdo, I., Medina, J.H., 2008. Reviews: BDNF and memory formation and storage. The Neuroscientist 14, 147-156.

Betts, K.S., Williams, G.M., Najman, J.M., Alati, R., 2015. The relationship between maternal depressive, anxious, and stress symptoms during pregnancy and adult offspring behavioral and emotional problems. Depression and Anxiety 32, 82-90.

Blaze, J., Asok, A., Roth, T.L., 2015. The long-term impact of adverse caregiving environments on epigenetic modifications and telomeres. Frontiers in Behavioral Neuroscience 9, 79.

Blaze, J., Roth, T.L., 2017. Caregiver maltreatment causes altered neuronal DNA methylation in female rodents. Development and Psychopathology In press.

Blaze, J., Scheuing, L., Roth, T.L., 2013. Differential methylation of genes in the medial prefrontal cortex of developing and adult rats following exposure to maltreatment or nurturing care during infancy. Developmental Neuroscience 35, 306-316.

Bock, J., Wainstock, T., Braun, K., Segal, M., 2015. Stress in utero: prenatal programming of brain plasticity and cognition. Biological Psychiatry 78, 315-326.

Boersma, G.J., Lee, R.S., Cordner, Z.A., Ewald, E.R., Purcell, R.H., Moghadam, A.A., Tamashiro, K.L., 2014. Prenatal stress decreases Bdnf expression and increases methylation of Bdnf exon IV in rats. Epigenetics 9, 437-447.

Botha, M., Grace, L., Bugarith, K., Russell, V.A., Kidd, M., Seedat, S., Hemmings, S.M., 2012. The impact of voluntary exercise on relative telomere length in a rat model of developmental stress. BMC Res Notes 5, 697. 
Bronson, S., Bale, T., 2014. Prenatal stress-induced increases in placental inflammation and offspring hyperactivity are male-specific and ameliorated by maternal antiinflammatory treatment. Endocrinology 155, 2635-2646.

Cao-Lei, L., Massart, R., Suderman, M.J., Machnes, Z., Elgbeili, G., Laplante, D.P., Szyf, M., King, S., 2014. DNA methylation signatures triggered by prenatal maternal stress exposure to a natural disaster: project Ice Storm. PLoS One 9, e107653.

Dabouras, V., Rothermel, A., Reininger-Mack, A., Wien, S.L., Layer, P.G., Robitzki, A.A., 2004. Exogenous application of glucose induces aging in rat cerebral oligodendrocytes as revealed by alteration in telomere length. Neuroscience Letters 368, 68-72.

Daviu, N., Rabasa, C., Nadal, R., Armario, A., 2014. Comparison of the effects of single and daily repeated immobilization stress on resting activity and heterotypic sensitization of the hypothalamic-pituitary-adrenal axis. Stress 17, 176-185.

de Souza, M.A., Centenaro, L.A., Menegotto, P.R., Henriques, T.P., Bonini, J., Achaval, M., Lucion, A.B., 2013. Prenatal stress produces social behavior deficits and alters the number of oxytocin and vasopressin neurons in adult rats. Neurochemical Research 38, 1479-1489.

Detich, N., Theberge, J., Szyf, M., 2002. Promoter-specific activation and demethylation by MBD2/demethylase. Journal of Biological Chemistry 277, 35791-35794.

Dincheva, I., Lynch, N., Lee, F., 2016. The role of BDNF in the development of fear learning. Depression and Anxiety 33, 907-916.

Dong, E., Dzitoyeva, S.G., Matrisciano, F., Tueting, P., Grayson, D.R., Guidotti, A., 2015. BDNF epigenetic modifications associated with schizophrenia-like phenotype induced by prenatal stress in mice. Biological Psychiatry 77.

Driscoll, C.A., Barr, C.S., 2016. Studying longitudinal trajectories in animal models of psychiatric illness and their translation to the human condition. Neuroscience Research 102, 6777.

Entringer, S., Epel, E.S., Kumsta, R., Lin, J., Hellhammer, D.H., Blackburn, E.H., Wüst, S., Wadhwa, P.D., 2011. Stress exposure in intrauterine life is associated with shorter telomere length in young adulthood. Proceedings of the National Academy of Sciences of the United States of America 108, E513-E518.

Entringer, S., Epel, E.S., Lin, J., Buss, C., Shahbaba, B., Blackburn, E.H., Simhan, H.N., Wadhwa, P.D., 2013. Maternal psychosocial stress during pregnancy is associated with newborn leukocyte telomere length. American Journal of Obstetrics and Gynecology 208, e7.

Flanary, B.E., Streit, W.J., 2003. Telomeres shorten with age in rat cerebellum and cortex in vivo. J Anti Aging Med 6, 299-308. 
Fuchikami, M., Morinobu, S., Segawa, M., Okamoto, Y., Yamawaki, S., Ozaki, N., Inoue, T., Kusumi, I., Koyama, T., Tsuchiyama, K., Terao, T., 2011. DNA methylation profiles of the brain-derived neurotrophic factor (BDNF) gene as a potent diagnostic biomarker in major depression. PLoS ONE 6, e23881.

Hoek, H., Susser, E., Buck, K., Lumey, L., Lin, S., Gorman, J., 1996. Schizoid personality disorder after prenatal exposure to famine. American Journal of Psychiatry 153, 1637-1639.

Jensen Peña, C., Monk, C., Champagne, F.A., 2012. Epigenetic effects of prenatal stress on $11 \beta$ Hydroxysteroid Dehydrogenase-2 in the placenta and fetal brain. PLoS One 7, e39791.

Kananen, L., Surakka, I., Pirkola, S., Suvisaari, J., Lonnqvist, J., Peltonen, L., Ripatti, S., Hovatta, I., 2010. Childhood adversities are associated with shorter telomere length at adult age both in individuals with an anxiety disorder and controls. PLoS One 5, e10826.

Keller, S., Sarchiapone, M., Zarrilli, F., et al., 2010. Increased BDNF promoter methylation in the Wernicke area of suicide subjects. Archives of General Psychiatry 67, 258-267.

Kiecolt-Glaser, J.K., Gouin, J.P., Weng, N.P., Malarkey, W.B., Beversdorf, D.Q., Glaser, R., 2011. Childhood adversity heightens the impact of later-life caregiving stress on telomere length and inflammation. Psychosomatic Medicine 73, 16-22.

Koenig, J.I., Elmer, G.I., Shepard, P.D., Lee, P.R., Mayo, C., Joy, B., Hercher, E., Brady, D.L., 2005. Prenatal exposure to a repeated variable stress paradigm elicits behavioral and neuroendocrinological changes in the adult offspring: potential relevance to schizophrenia. Behavioural Brain Research 156, 251-261.

Kohli, R.M., Zhang, Y., 2013. TET enzymes, TDG and the dynamics of DNA demethylation. Nature 502, 472-479.

Kundakovic, M., Champagne, F.A., 2011. Epigenetic perspective on the developmental effects of bisphenol A. Brain, Behavior, and Immunity 25, 1084-1093.

Kundakovic, M., Lim, S., Gudsnuk, K., Champagne, F.A., 2013. Sex-specific and straindependent effects of early life adversity on behavioral and epigenetic outcomes. Frontiers in Psychiatry 4.

Leventopoulos, M., Rüedi-Bettschen, D., Knuesel, I., Feldon, J., Pryce, C.R., Opacka-Juffry, J., 2007. Long-term effects of early life deprivation on brain glia in Fischer rats. Brain Research $1142,119-126$.

Linkus, B., Wiesner, D., Messner, M., Karabatsiakis, A., Scheffold, A., Rudolph, K.L., Thal, D.R., Weishaupt, J.H., Ludolph, A.C., Danzer, K.M., 2016. Telomere shortening leads to earlier age of onset in ALS mice. Aging 8, 382-393. 
Livak, K.J., Schmittgen, T.D., 2001. Analysis of relative gene expression data using real-time quantitative PCR and the 2- $\Delta \Delta \mathrm{CT}$ method. Methods 25, 402-408.

Lubin, F.D., Roth, T.L., Sweatt, J.D., 2008. Epigenetic regulation of bdnf gene transcription in the consolidation of fear memory. Journal of Neuroscience 28, 10576-10586.

Lupien, S.J., McEwen, B.S., Gunnar, M.R., Heim, C., 2009. Effects of stress throughout the lifespan on the brain, behaviour and cognition. Nature Reviews Neuroscience 10, 434-445.

Ma, D.K., Jang, M.H., Guo, J.U., Kitabatake, Y., Chang, M.L., Pow-Anpongkul, N., Flavell, R.A., Lu, B., Ming, G.L., Song, H., 2009. Neuronal activity-induced Gadd45b promotes epigenetic DNA demethylation and adult neurogenesis. Science 323, 1074-1077.

Malaspina, D., Corcoran, C., Kleinhaus, K., Perrin, M., Fennig, S., Nahon, D., Friedlander, Y., Harlap, S., 2008. Acute maternal stress in pregnancy and schizophrenia in offspring: a cohort prospective study. BMC Psychiatry 8, 71.

Marchetto, N.M., Glynn, R.A., Ferry, M.L., Ostojic, M., Wolff, S.M., Yao, R., Haussmann, M.F., 2016. Prenatal stress and newborn telomere length. American Journal of Obstetrics and Gynecology 215, 94.e91-94.e98.

Martinowich, K., Manji, H., Lu, B., 2007. New insights into BDNF function in depression and anxiety. Nature Neuroscience 10, 1089-1093.

Matrisciano, F., Tueting, P., Dalal, I., Kadriu, B., Grayson, D.R., Davis, J.M., Nicoletti, F., Guidotti, A., 2013. Epigenetic modifications of GABAergic interneurons are associated with the schizophrenia-like phenotype induced by prenatal stress in mice. Neuropharmacology 68, 184194.

McEwen, B.S., Bowles, N.P., Gray, J.D., Hill, M.N., Hunter, R.G., Karatsoreos, I.N., Nasca, C., 2015. Mechanisms of stress in the brain. Nat Neurosci 18, 1353-1363.

Morgan, C.P., Bale, T.L., 2011. Early prenatal stress epigenetically programs dysmasculinization in second-generation offspring via the paternal lineage. Journal of Neuroscience 31, 1174811755.

Mueller, B.R., Bale, T.L., 2007. Early prenatal stress impact on coping strategies and learning performance is sex dependent. Physiology and Behavior 91, 55-65.

Mueller, B.R., Bale, T.L., 2008. Sex-specific programming of offspring emotionality after stress early in pregnancy. Journal of Neuroscience 28, 9055-9065.

Nye, M.D., Fry, R.C., Hoyo, C., Murphy, S.K., 2014. Investigating epigenetic effects of prenatal exposure to toxic metals in newborns: challenges and benefits. Medical Epigenetics 2, 53-59. O'Callaghan, N.J., Fenech, M., 2011. A quantitative PCR method for measuring absolute telomere length. Biological Procedures Online 13, 3. 
O'Donovan, A., Epel, E., Lin, J., Wolkowitz, O., Cohen, B., Maguen, S., Metzler, T., Lenoci, M., Blackburn, E., Neylan, T.C., 2011. Childhood trauma associated with short leukocyte telomere length in posttraumatic stress disorder. Biological Psychiatry 70, 465-471.

Painter, R.C., Osmond, C., Gluckman, P., Hanson, M., Phillips, D.I.W., Roseboom, T.J., 2008. Transgenerational effects of prenatal exposure to the Dutch famine on neonatal adiposity and health in later life. BJOG: An International Journal of Obstetrics and Gynaecology 115, 12431249.

Perroud, N., Salzmann, A., Prada, P., Nicastro, R., Hoeppli, M.E., Furrer, S., Ardu, S., Krejci, I., Karege, F., Malafosse, A., 2013. Response to psychotherapy in borderline personality disorder and methylation status of the BDNF gene. Translational Psychiatry 3, e207.

Roseboom, T.J., Painter, R.C., van Abeelen, A.F.M., Veenendaal, M.V.E., de Rooij, S.R., 2011. Hungry in the womb: what are the consequences? Lessons from the Dutch famine. Maturitas 70, 141-145.

Roth, T.L., Lubin, F.D., Funk, A.J., Sweatt, J.D., 2009. Lasting epigenetic influence of early-life adversity on the BDNF gene. Biological Psychiatry 65, 760-769.

Roth, T.L., Matt, S., Chen, K., Blaze, J., 2014. Bdnf DNA methylation modifications in the hippocampus and amygdala of male and female rats exposed to different caregiving environments outside the homecage. Developmental Psychobiology 56, 1755-1763.

Roth, T.L., Zoladz, P.R., Sweatt, J.D., Diamond, D.M., 2011. Epigenetic modification of hippocampal Bdnf DNA in adult rats in an animal model of post-traumatic stress disorder. Journal of Psychiatric Research 45, 919-926.

Schuurmans, C., Kurrasch, D.M., 2013. Neurodevelopmental consequences of maternal distress: what do we really know? Clinical Genetics 83, 108-117.

Shalev, I., Caspi, A., Ambler, A., Belsky, D.W., Chapple, S., Cohen, H.J., Israel, S., Poulton, R., Ramrakha, S., Rivera, C.D., Sugden, K., Williams, B., Wolke, D., Moffitt, T.E., 2014a. Perinatal complications and aging indicators by midlife. Pediatrics 134, e1315-e1323.

Shalev, I., Moffitt, T.E., Braithwaite, A.W., Danese, A., Fleming, N.I., Goldman-Mellor, S., Harrington, H., Houts, R.M., Israel, S., Poulton, R., Robertson, S.P., Sugden, K., Williams, B., Caspi, A., 2014b. Internalizing disorders and leukocyte telomere erosion: A prospective study of depression, generalized anxiety disorder and post-traumatic stress disorder. Molecular psychiatry 19, 1163-1170.

Sickmann, H.M., Arentzen, T.S., Dyrby, T.B., Plath, N., Kristensen, M.P., 2015. Prenatal stress produces sex-specific changes in depression-like behavior in rats: implications for increased vulnerability in females. Journal of Developmental Origins of Health and Disease 6, 462-474. 
Slagboom, P.E., Droog, S., Boomsma, D.I., 1994. Genetic determination of telomere size in humans: a twin study of three age groups. American journal of human genetics 55, 876.

Smith, A.K., Conneely, K.N., Kilaru, V., Mercer, K.B., Weiss, T.E., Bradley, B., Tang, Y., Gillespie, C.F., Cubells, J.F., Ressler, K.J., 2011. Differential immune system DNA methylation and cytokine regulation in post-traumatic stress disorder. American Journal of Medical Genetics Part B: Neuropsychiatric Genetics 156, 700-708.

Soztutar, E., Colak, E., Ulupinar, E., 2016. Gender- and anxiety level-dependent effects of perinatal stress exposure on medial prefrontal cortex. Experimental Neurology 275, Part 2, 274284.

St-Cyr, S., McGowan, P.O., 2015. Programming of stress-related behavior and epigenetic neural gene regulation in mice offspring through maternal exposure to predator odor. Frontiers in Behavioral Neuroscience 9, 145.

Szebeni, A., Szebeni, K., DiPeri, T., Chandley, M.J., Crawford, J.D., Stockmeier, C.A., Ordway, G.A., 2014. Shortened telomere length in white matter oligodendrocytes in major depression: potential role of oxidative stress. Int J Neuropsychopharmacol 17, 1579-1589.

Thomas, P., O' Callaghan, N.J., Fenech, M., 2008. Telomere length in white blood cells, buccal cells and brain tissue and its variation with ageing and Alzheimer's disease. Mechanisms of Ageing and Development 129, 183-190.

Tyrka, A.R., Price, L.H., Kao, H.T., Porton, B., Marsella, S.A., Carpenter, L.L., 2010. Childhood maltreatment and telomere shortening: preliminary support for an effect of early stress on cellular aging. Biological Psychiatry 67, 531-534.

Wei, Y.B., Backlund, L., Wegener, G., Mathe, A.A., Lavebratt, C., 2015. Telomerase dysregulation in the hippocampus of a rat model of depression: normalization by lithium. Int $\mathbf{J}$ Neuropsychopharmacol 18, pyv002.

Wilson, C.A., Schade, R., Terry Jr, A.V., 2012. Variable prenatal stress results in impairments of sustained attention and inhibitory response control in a 5-choice serial reaction time task in rats. Neuroscience 218, 126-137.

Wilson, C.A., Vazdarjanova, A., Terry Jr, A.V., 2013. Exposure to variable prenatal stress in rats: Effects on anxiety-related behaviors, innate and contextual fear, and fear extinction. Behavioural Brain Research 238, 279-288.

Zeichner, S.L., Palumbo, P., Feng, Y., Xiao, X., Gee, D., Sleasman, J., Goodenow, M., Biggar, R., Dimitrov, D., 1999. Rapid telomere shortening in children. Blood 93, 2824-2830. 


\section{Figure Captions}

Figure 1. Bdnf IV DNA methylation in the A) medial prefrontal cortex and B) ventral hippocampus. UVPS male offspring had significantly higher methylation compared to control males and UVPS females across most CG sites within the medial prefrontal cortex. ${ }^{*} \mathrm{p}<0.05$, $* * \mathrm{p}<0.01, * * * \mathrm{p}<0.001 ; \mathrm{n}=6-7 / \mathrm{sex} /$ condition; error bars represent SEM.

Figure 2. Telomere length in the adult medial prefrontal cortex. Males who experienced UVPS had significantly shorter telomeres compared to control males. UVPS females also exhibited marginally shorter telomeres relative to controls. ${ }^{*} \mathrm{p}<0.05 ; \mathrm{n}=6-7 / \mathrm{sex} /$ condition; error bars represent SEM. 


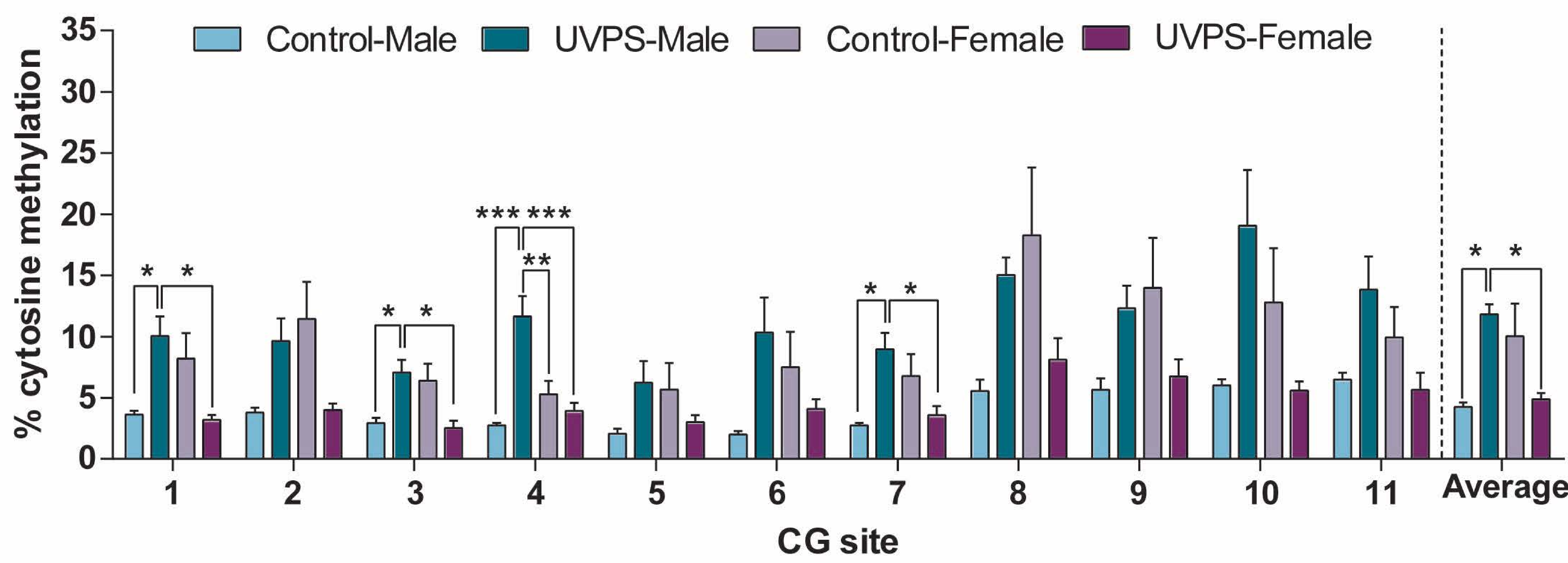

B)




